\section{Developmental trends in the use of logical connectives}

\author{
DENISE SHINE and JOHN F. WALSH \\ Fordham University, New York, N.Y. 10458
}

Children in Grades 2, 4, 6, and 8 were tested on their ability to comprehend and categorize stimuli involving eight logical connectives: conjunction, disjunction, conditional, biconditional, and their negatives. Three distinct groupings of difficulty were apparent. The easy group of connectives (negative implication, negative disjunction, and conjunction) had the common factor of being verbalized with the connective "and." The second group, showing a definite developmental trend, consisted of the negative biconditional and the disjunctive, which shared the common factor of being verbalized with the connective "or." The third group revealed an almost total failure to understand implication and biconditional, verbalized with "if," as well as difficulty in handling the negative conjunctive which verbalizes as a disjunction of negatives. Results were considered to support Bourne's hypothesis of an intuitive truth-table approach to multiple rule learning rather than Neisser and Weene's postulation of a hierarchy of difficulty levels.

Piaget's emphasis on the role of logical structures in the intellectual growth of the child has stimulated much research on the use of symbolic logic in cognitive learning (Bourne, 1966, 1967; Bower \& King, 1967; Haygood \& Bourne, 1965; King, 1966, 1968). Neisser \& Weene (1962) have proposed progressively more difficult hierarchies in concept attainment at three structurally different levels of complexity, with Level I consisting of two univeriate concepts, Level II, six bivariate concepts, and Level III, two complex concepts formed by disjunction of conjunctive pairs. The increasing ability of children to learn and use logical connectives with advances in age was related by Youniss \& Furth (1964) to Piaget's distinction between concrete and formal operational developmental stages. However, only three Level II concepts (conjunction, exclusive disjunction, and conjunctive absence) were employed by Youniss and Furth, and results were not clearcut. In a recent report, Bourne (1969) suggested that multiple rule learning can be best described as an intuitive version of the logical truth-table, whereby the $S$ learns to mediate the stimulus pattern to response category assignments by collapsing and coding the entire stimulus population into four classes: TT, TF, FT, and FF. Once the coding process has been completed, each new learning problem is solved by learning the connections between the four coded classes of patterns and the two response categories. Such coding of stimulus classes is apparently encouraged by enforced attention to relevant attributes (Johnson, Warner, \& Lee, 1970), for example by forced verbalization to limit attention (King, \& Holt, 1970). King (1968) rejected the hypothesis that encoding difficulty Four logical connectives (and, or, if, only if) and their negations were tested, each of the eight being presented on three different items for a total of 24 trials. Each item consisted of four symbols varying along two dimensions-different for each item. The three types of items used with the relevant dimensions are shown in Fig. 1.

Each of the eight logical connectives was used once with the three item types, presentation of connectives and item types being ordered randomly. Ss were given a paper containing the 24 randomly ordered items, and statements were presented orally. For example, one disjunctive statement was given as: "It is big, or, it is a circle," the other two changing the relevant dimension to match the stimuli being presented. Preliminary instructions included examples involving other combinations of dimensions than those presented in the test. Directions were to cross out the figures for which the statement was true, whether this was none, one, two, three, or all of the figures. Since 16 different answers were possible to each question with only one correct, the effect of guessing was minimal. Children were urged to try to understand the statement before marking the answer. The number of correct answers for each of the eight logical connective sets was used as $S$ scores.

\section{RESULTS}

All children were able to answer at least some of the items correctly but no one was able to answer all correctly. Scores were submitted to an overall analysis of variance. Significant differences were found for grade level $[F(3,119)=12.2, p<.01]$, type of connective $[F(7,840)=447.2$, $p<.01]$, and their interaction $[F(21,840)=5.2, p<.01]$. Since sex differences were insignificant, boys' and girls' scores were combined for further analysis. The differentiation exhibited by the eight types of connectives is most clearly shown in Fig. 2. Where two symbolic statements are given, the statement was verbalized as indicated by the second statement.

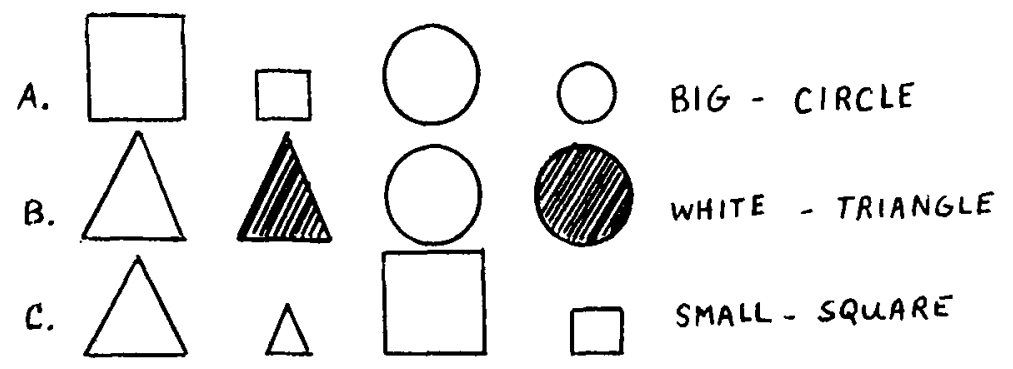

Fig. 1. Items and revelant dimensions. 


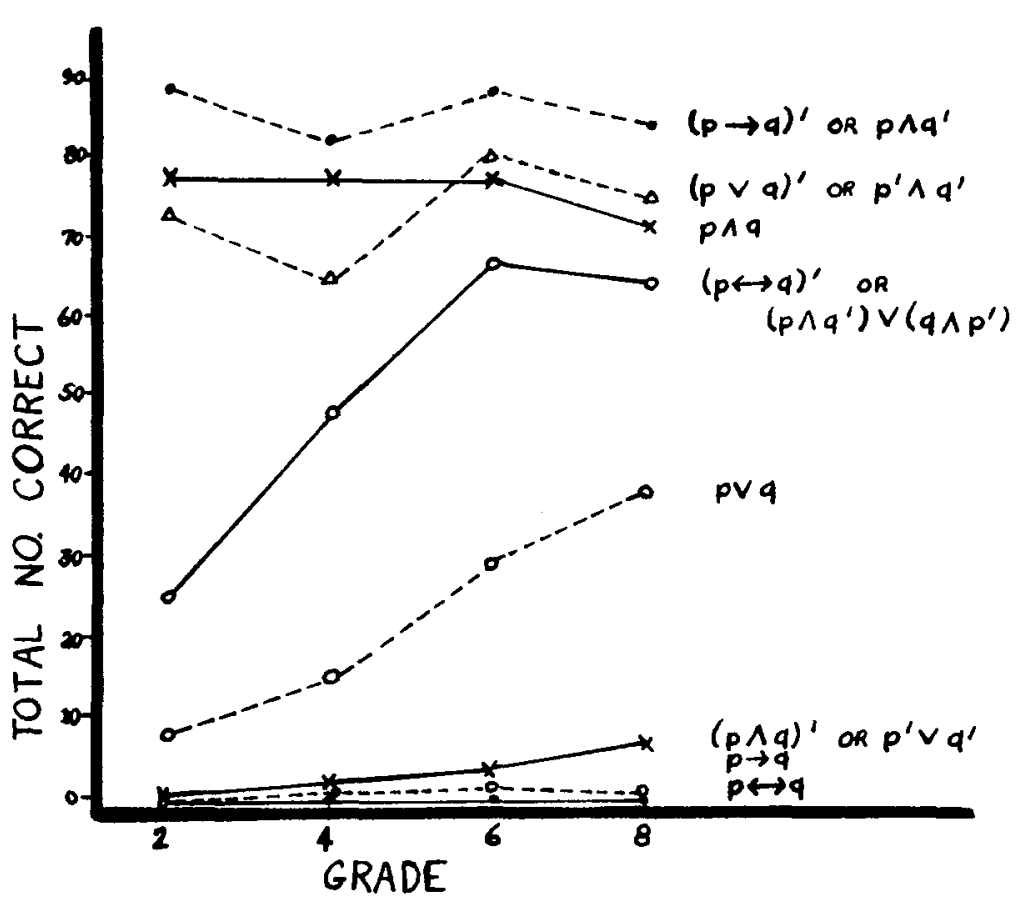

Fig. 2. Correct responses at each grade to the type of connective.

For example, the negation of the implication, $(p \rightarrow q)^{\prime}$, was verbalized as its logical equivalent, $p \wedge q^{\prime}$ (e.g., It is big and it is not a circle).

Three groupings of difficulty are apparent from the graph. An easy group of connectives (negative implication, negative disjunction, and conjunction), with little difference in difficulty from grade to grade, has the common factor of being verbalized with the connective "and." The second group, showing a definite developmental trend, consisted of two connectives, the negative biconditional and the disjunctive, which share the common factor of being verbalized with the connective "or." The third group reveals an almost total failure to understand implication and biconditional, both verbalized (in this study) with the word "if," as well as a slight increase with age in the ability to handle the negative conjunctive which verbalizes as a disjunction of negatives. A further analysis of the types of disjunctive errors made reveals a preponderance at each level of errors giving a conjunction of attributes or the conjunction plus one of the alternates of the disjunctive pair. Diffuse errors, or errors without any apparent reasoning process, tended to decrease with age.

$$
\text { DISCUSSION }
$$

The most striking result of this study is the strong developmental trend shown in the ability to comprehend and use the disjunctive connective regardless of the complexity of the units involved.
Improvement is especially noticeable at the sixth grade, the age group where Piaget postulates the acquisition of formal operations. Contrary to Neisser and Weene's hierarchy, the negative biconditional (verbalized as " $p$ and not $q$, or, q and not p"), a Level III concept, as considerably easier than several Level II concepts for all children. The fact that the easiest concept for all, the negative conditional (verbalized as "p and not q"), constituted each of the units for the negative biconditional gives considerable support to Bourne's hypothesis of an intuitive truth-table approach. By collapsing over each easy unit and connecting them with "or," the relationship was more accurately perceived than even the straight disjunctive. However, it is important to note that the negative biconditional forms an exclusive disjunction without the possibility of both units simultaneously, whereas the ordinary disjunction was nonexclusive. Still, an analysis of the errors made in the latter case showed almost no errors because of an exclusive interpretation. The results would suggest that difficulty is more qualitative than quantitative and does not increase by the linear stringing together of simpler units but by the inability to collapse over and encode entire configurations of stimuli.

The total failure of the children to comprehend the conditional and biconditional points up the necessity for educators to include some training in logical relationships.
In a culture where implications are embedded in everyday life, children should develop critical abilities in dealing with the dangers of judging on false premises. Presumably, if logically equivalent forms had been used ("not $p$ or $q$ " in place of "if $p$ then $q$ "), results would have been more intermediate, but the deficit in understanding and the primitive equation of implication and conjunction, even among older children, would not have been apparent. Indeed, the pervading influence of the conjunctive in the minds of children and their tendency to combine any two things in juxtaposition show the necessity for better training in such discrimination to adapt to a world heavily inclined to "either/or" and "if" rather than the simple "and."
BOURNE, L. E., JR. Human conceptual behavior. Boston: Allyn \& Bacon, 1966. BOURNE, L. E., JR. Learning and utilization of conceptual rules. In B. Kleinmuntz (Ed.), Concepts and the structure of memory. New York: Wiley, 1967.

BOURNE, L. E., JR: Development of conceptual skills: Some preliminary findings. Technical Report No.81. Wisconsin Research and Development Center for Cognitive Learning. University of Wisconsin, Madison, Wis., March 1969.

BOWER, A. C., \& KING, W. L. The effect of number of irrelevant stimulus dimensions, verbalization, and scx on learning biconditional classification rules. Psychonomic Science, 1967, 8, 453-454.

HAYGOOD, R. C., \& BOURNE, L. E., JR. Attribute- and rule-learning aspects of conceptual behavior. Psychological Review, 1965, 72, 179-195.

JOHNSON, P. J., WARNER, M. S., \& LEE, D. R. Effects of enforced attention and stimulus phasing upon rule learning in children. Journal of Experimental Child Psycholgy, 1970, 9, 388-399.

KING, W. $\dot{L}$. Learning and utilization of conjunctive and disiunctive classification rules: A developmental study. Journal of Experimental Child Psychology, 1966, 4, 217-231.

KING, W. $L$. Rule learning and transfer as a function of age and stimulus structure. Child Development, 1968, 39, 311-324.

KING, W. L. \& HOLT, J. R. Conjunctive and disjunctive rule learning as a function of age and forced verbalization. Journal of Experimental Child Psychology, 1970. 10, 100-111.

NEISSER, U., \& WEENE, P. Hierarchies in concept attainment. Journal of Experimental Psychology, 1962, 64, 640-645.

YOUNISS, J., \& FURTH, H. G. Attainment and transfer of logical connectives in children. Journal of Educational Psychology, 1964, 55, 357-361. 